\title{
Imaging Performance Analysis of an EO/IR Dual Band Airbome Camera
}

\author{
Jun Ho Lee*, Yong-Suk Jung, Seung-Yeol Ryoo, and Young-Ju Kim \\ Department of Optical Engineering, Kongju National University, Kongju 314-701, Korea \\ Byong-Ug Park, Hyun-Jung Kim, and Sung-Kie Youn \\ Department of Mechanical Engineering, KAIST, Daejon 305-701, Korea \\ Kwang-Woo Park and Haeng Bok Lee \\ Agency for Defense Development, P.O. Box 35, Daejon 305-600, Korea
}

(Received February 23, 2011 : accepted March 31, 2011)

\begin{abstract}
An airborne sensor is developed for remote sensing on an aerial vehicle (UV). The sensor is an optical payload for an eletro-optical/infrared (EO/IR) dual band camera that combines visible and IR imaging capabilities in a compact and lightweight package. It adopts a Ritchey-Chrétien telescope for the common front end optics with several relay optics that divide and deliver EO and IR bands to a charge-coupleddevice (CCD) and an IR detector, respectively. The EO/IR camera for dual bands is mounted on a two-axis gimbal that provides stabilized imaging and precision pointing in both the along and cross-track directions. We first investigate the mechanical deformations, displacements and stress of the EO/IR camera through finite element analysis (FEA) for five cases: three gravitational effects and two thermal conditions. For investigating gravitational effects, one gravitational acceleration $(1 \mathrm{~g})$ is given along each of the $+x$, $+y$ and $+\mathrm{z}$ directions. The two thermal conditions are the overall temperature change to $30^{\circ} \mathrm{C}$ from $20^{\circ} \mathrm{C}$ and the temperature gradient across the primary mirror pupil from $-5^{\circ} \mathrm{C}$ to $+5^{\circ} \mathrm{C}$. Optical performance, represented by the modulation transfer function (MTF), is then predicted by integrating the FEA results into optics design/analysis software. This analysis shows the IR channel can sustain imaging performance as good as designed, i.e., MTF 38\% at 13 line-pairs-per-mm (lpm), with refocus capability. Similarly, the EO channel can keep the designed performance (MTF 73\% at $27.3 \mathrm{lpm}$ ) except in the case of the overall temperature change, in which the EO channel experiences slight performance degradation (MTF $16 \%$ drop) for $20^{\circ} \mathrm{C}$ overall temperate change.
\end{abstract}

Keywords: MTF, Airborne sensor, Optomechanics, EO, IR

OCIS codes : (120.4880) Optomechanics; (120.4820) Optical systems; (280.4788) Optical sensing and sensors

\section{INTRODUCTION}

Satellites or aircrafts have been used for remote sensing for many years. The most common payloads for both spaceborne and air-borne remote sensing are optical cameras or telescopes, which form images (of Earth's surface) on image detectors [1-4]. The most common detectors for optical imaging are charge coupled devices (CCDs) and infrared detectors [5-8]. The distinction between the two is working wavelength or spectral quantum efficiency: A CCD can sense light in the near ultraviolet (UV) down to near IR, which is often called the electro-optic (EO) spectral band, and IR detectors can sense the IR spectrum. Fig. 1 shows a typical spectral response of a CCD (e2V AT71201M [6]) and a mid-wave HgCdTe IR detector [8].

An airborne sensor is developed for remote sensing on an aerial vehicle (AV). The sensor is an optical payload for an electro-optical/infrared (EO/IR) dual band camera that

\footnotetext{
*Corresponding author: jhlsat@kongju.ac.kr

Color versions of one or more of the figures in this paper are available online.
} 
combines visible and IR imaging capabilities in a compact and lightweight manner, as similarly reported by the previous studies [2, 7, 9-11]. It adopts a Ritchey-Chrétien telescope for the common front end optics with several relay optics that divide and deliver the EO and IR bands to a CCD and an IR detector, respectively. The EO/IR camera for dual bands is mounted on a two-axis gimbal that provides stabilized imaging and precision pointing in both the along and crosstrack directions. Fig. 2 shows a schematic of the expanded view of the EO/IR dual band camera.

The UV payload should be designed to sustain its imaging requirements over hostile environments, such as temperature change between on-ground and in-air at its flying altitude, gravity acceleration due to the UAV's maneu-

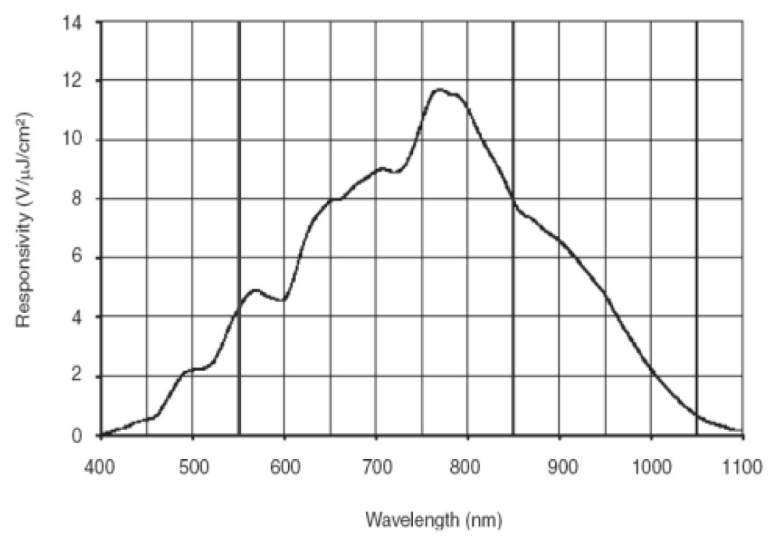

(a) CCD (e2V AT71201M [6])

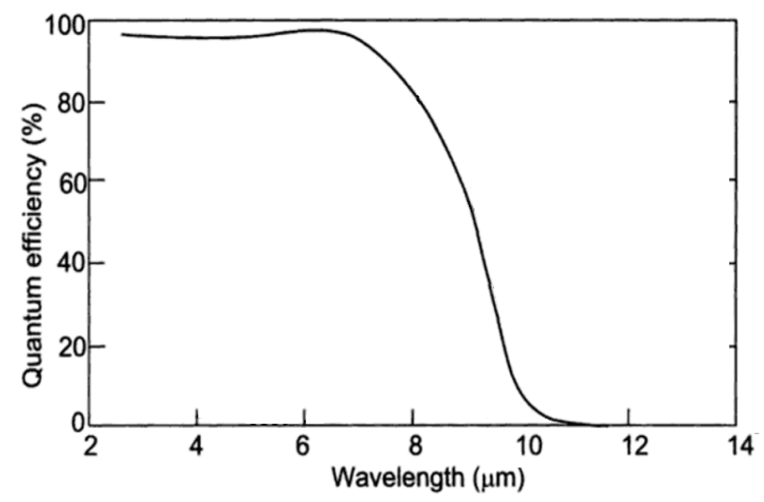

(b) IR detector (HgCdTe [8])

FIG. 1. Spectral response of the two most common imaging detectors for remote sensing; (a) CCD and(b) IR detector.

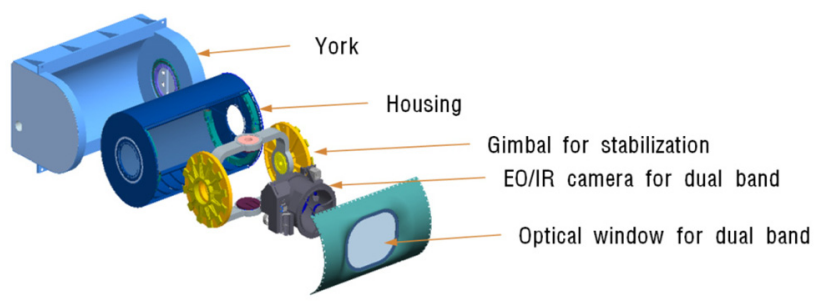

FIG. 2. Expanded view of the EO/IR dual band sensor. vering, and jitter movement caused by the AV propulsion [12]. In addition, stringent constraints are imposed on the weight and volume of the payload design.

We first investigate the mechanical deformations, displacements and stress of the EO/IR camera through finite element analysis (FEA). The optical performance, represented by the modulation transfer function (MTF), is then predicted by integrating the FEA results into optics design/analysis software (ZEMAX) [13]. The analysis is done for gravity and thermoelastic deformations.

\section{FEA MODEL \& ANALYSIS}

\subsection{Optical Model}

The EO/IR dual band camera is basically a Ritchey-Chrétien telescope consisting of a primary mirror and a secondary mirror. The entrance pupil of the camera is about $30 \mathrm{~cm}$ in diameter. The telescope is then followed by a beam splitter which splits the incoming beam to EO and IR channels. Each beam is then forwarded by relay optics to its corresponding detector. Fig. 3 shows the schematic optical layout of the camera. Table 1 lists the primary parameters of the EO and IR channels.

\subsection{FEA Model and Boundary Conditions}

In order to calculate the surface deformation and displacements precisely, a FEA model of the EO/IR camera is expressed in the commercial software I-DEAS. Fig. 4 shows the isometric and bottom views of the FEA model. The boundary conditions of the model are set by fixing the locations of the gimbal mounts, which are expressed as dotted circles in Fig. 4. The directions of the three axes that are referred to later in this paper are also drawn in Fig. 4.

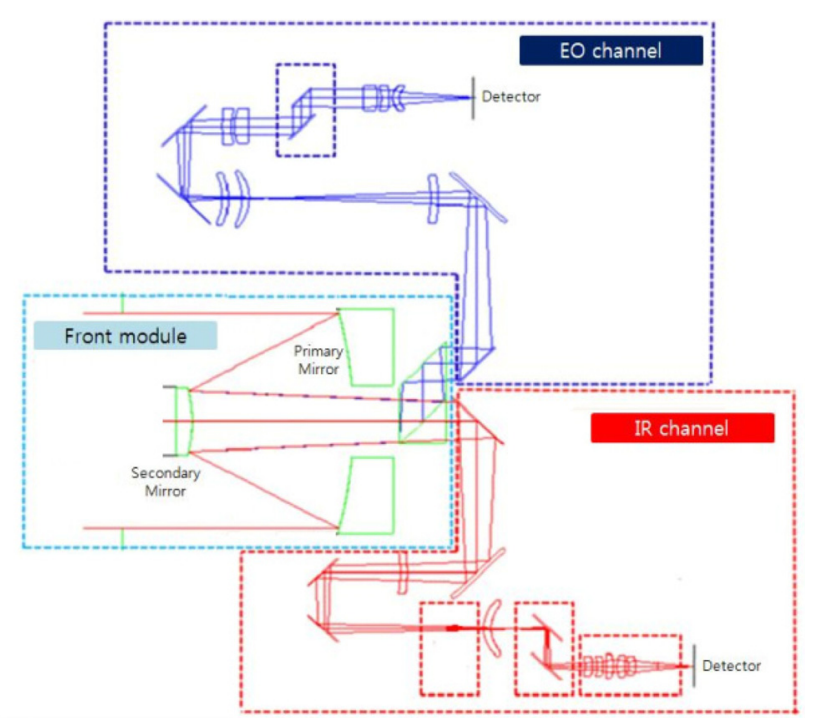

FIG. 3. Schematic optical layout of the EO/IR dual band IR camera. 
TABLE 1. Principal parameters of the EO and IR channels

\begin{tabular}{c|c|c}
\hline \hline Parameter & Electro-optic & IR \\
\hline Spectral range (or wavelength) & Visible & Mid IR \\
\hline Field of view (diagonal FOV) & $\pm 0.596^{\circ}$ & $1539 \mathrm{~mm}$ \\
\hline Focal length & $2112 \mathrm{~mm}$ & 5.6 \\
\hline F number & 7.4 & IR detector \\
\hline Focal plane type & CCD & $8 \times 8 \mathrm{~mm}$ \\
\hline Image size & $11 \times 11 \mathrm{~mm}$ &
\end{tabular}

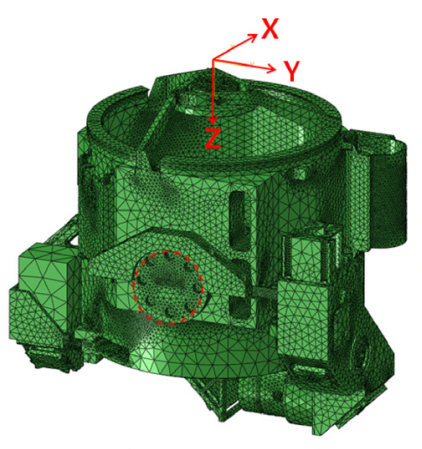

(a) Isometric view

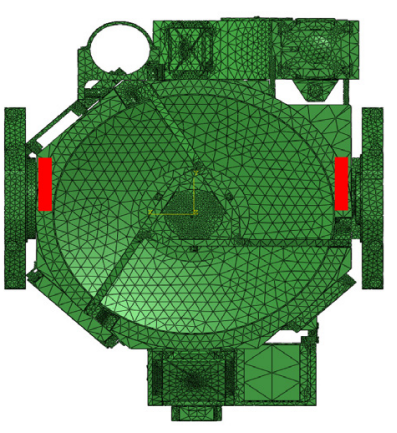

(b) Bottom view

FIG. 4. FEA model of the EO/IR camera.

\subsection{Analysis}

The analysis is performed for five cases: three gravitational effects and two thermal conditions. For investigating gravitational effects, one gravitational acceleration $(1 \mathrm{~g})$ is given along each of the $+\mathrm{X},+\mathrm{Y}$ and $+\mathrm{Z}$ directions. The two thermal conditions are the overall temperature change to $30^{\circ} \mathrm{C}$ from $20^{\circ} \mathrm{C}$ and the temperature gradient across the y axis from $-5^{\circ} \mathrm{C}$ to $+5^{\circ} \mathrm{C}$.

The FEA model is solved by a commercial FEA solver called ABAQUS. The optical surface deformation is then analyzed using the Zernike polynomial decomposition method $[14,15]$. A wavefront distortion $\phi(r, \theta)$ over a circular aperture of an arbitrary radius $R$ is expanded as a linear sum of Zernike polynomials $Z_{i}(\rho, \theta)$ given by

with $\rho=\mathrm{r} / \mathrm{R}$ and the coefficients $\alpha_{i}$ being given by

$$
a_{i}=\int d^{2} r \rho W(\rho) \phi(R \rho, \theta) Z_{i}(\rho, \theta),
$$

where the weighting function $W(\rho)$ defining the unit circular aperture is given by

$$
W(\rho)=\left\{\begin{array}{cc}
1 / \pi, & \rho \leq 1 \\
0, & \rho>1
\end{array}\right.
$$

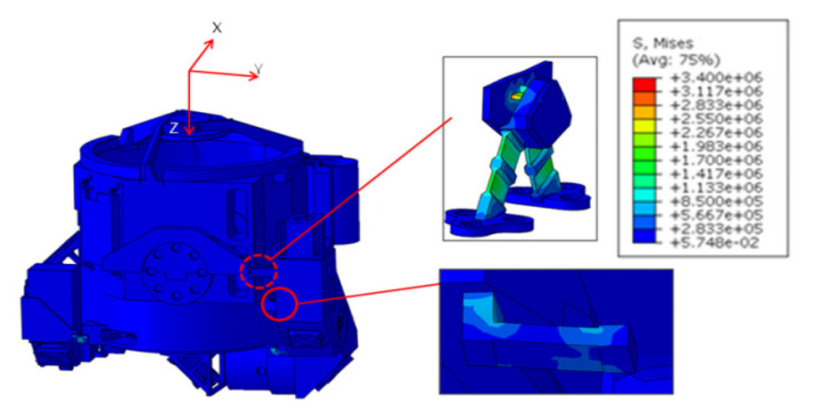

FIG. 5. Stress due to $+x$ gravity.

TABLE 2. Deformations of the primary and secondary mirrors due to $1 \mathrm{~g}$ gravitational force.

\begin{tabular}{c|c|c|c|c}
\hline \multirow{2}{*}{$\begin{array}{c}\text { Gravity } \\
\text { direction }\end{array}$} & \multicolumn{3}{|c}{ RMS Deformation Error (nm) } \\
\cline { 2 - 5 } & Primary mirror & \multicolumn{2}{c}{ Secondary mirror } \\
\cline { 2 - 5 } & $\begin{array}{c}\text { Without } \\
\text { RBM }\end{array}$ & $\begin{array}{c}\text { With } \\
\text { RBM }\end{array}$ & $\begin{array}{c}\text { Without } \\
\text { RBM }\end{array}$ & $\begin{array}{c}\text { With } \\
\text { RBM }\end{array}$ \\
\hline $\mathrm{X}$ & 4.17 & 8.24 & 0.27 & 78.33 \\
\hline $\mathrm{Y}$ & 4.08 & 20.31 & 0.27 & 72.7 \\
\hline $\mathrm{Z}$ & 16.21 & 406.15 & 0.88 & 315.04 \\
\hline
\end{tabular}

The first three Zernike polynomials are called the piston, the tip and the tilt. Since those terms represent the rigid-body motion (RBM) of a lens or mirror, those terms are often removed prior to optical calculations such as peak-to-valley (PV) and root-mean-squared (RMS) wavefront errors of a single optical surface.

\section{FEA RESULTS}

\subsection{Gravitational Effects}

The first investigation checks the structural stiffness. Fig. 5 shows the stress due to $1 \mathrm{~g}$ gravity along the $+\mathrm{X}$ direction. The maximum stress, which occurred at the flexure mount, is about $3.4 \mathrm{MPa}$, which is quite far away from the yield stress of the flexure material (invar), i.e., 145 Gpa. The FEA analysis also confirms the mechanical stiffness of the model over the $+\mathrm{Y}$ and $+\mathrm{Z}$ gravity.

Table 2 summarizes the deformations of the primary and 


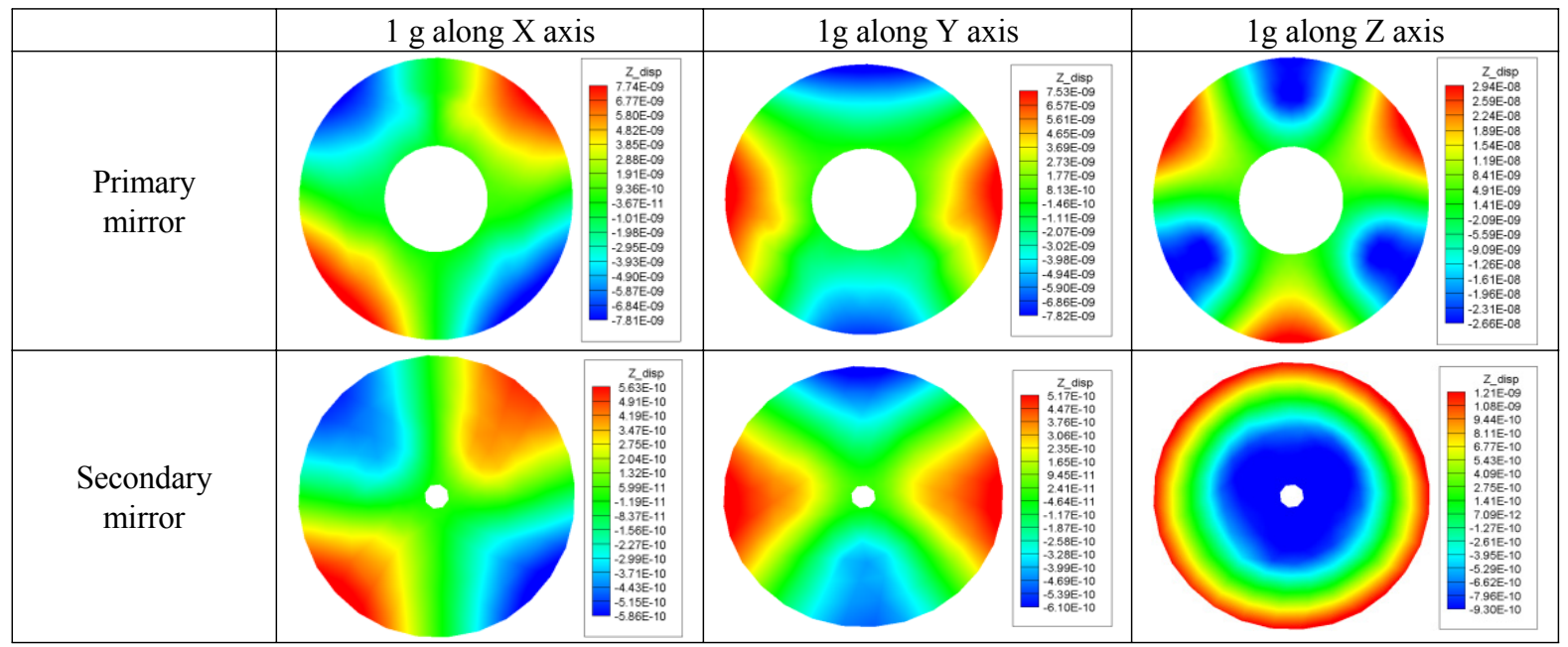

FIG. 6. Contour plots of the primary and secondary mirror deformations due to gravitational effects. Rigid body motion removed. Contour maps are in meter scale.

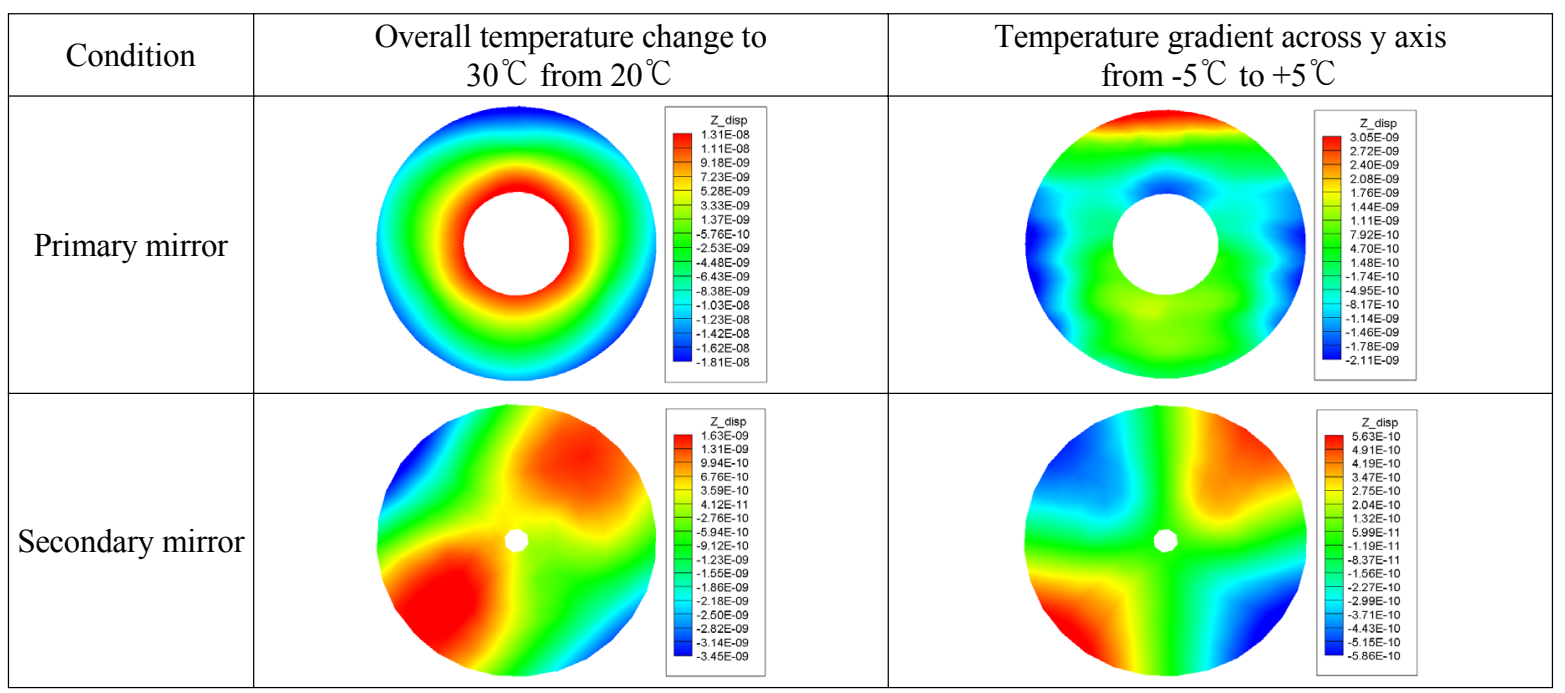

FIG. 7. Contour plots of the primary and secondary mirror deformations due to overall temperature rise and gradient across the $\mathrm{Y}$ axis. Rigid body motion removed. Contour maps are in meter scale.

secondary mirrors. The primary mirror is found to be more vulnerable to gravity. Fig. 6 shows the surface deformations of the primary and secondary mirrors with the rigid body motion removed.

\subsection{Thermal Effects}

The thermal effects are investigated for two conditions: a) the overall temperature change to $30^{\circ} \mathrm{C}$ from $20^{\circ} \mathrm{C}$ and b) the temperature gradient across the $\mathrm{Y}$ axis from $-5^{\circ} \mathrm{C}$ to $+5^{\circ} \mathrm{C}$. The analysis shows that the maximum stress is smaller than the yield strength of the material for all thermal conditions. Fig. 8 shows the stress due to the overall temperature change. Fig. 7 shows the contour plots of the primary and secondary mirrors for the two thermal conditions. The RMS displacements of the primary and secondary mirrors are

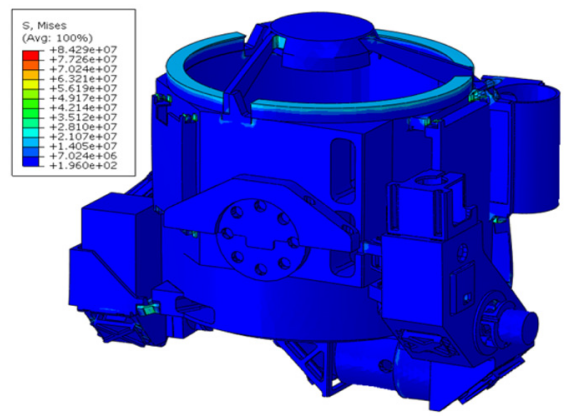

FIG. 8. Stress due to overall temperature change to $30^{\circ} \mathrm{C}$ from $20^{\circ} \mathrm{C}$.

9.1 and $1.11 \mathrm{~nm}$ for the overall temperature change, and 1.12 and $0.23 \mathrm{~nm}$ for the temperature gradient condition. 


\section{IMAGING PERFORMANCE PREDICTION}

\subsection{Method}

Conventionally FEA results are used to check whether the designed tolerances are satisfied [16]. This traditional approach is a top-down process which considers how simultaneous changes in multiple parameters interact in a statistical way using the Monte Carlo approach [17]. The Monte Carlo approach determines an optimum set of tolerance for a given optical product by taking into account the manufacturing and assembly variations that occur in mass production. We investigate optical performance by integrating the FEA results into optics design/analysis software. This way predicts the imaging performance of the camera more precisely than statistical prediction.

Most lens design and analysis software can import optical surface deformations, which are from FEA analysis or metro- logical measurement, into a lens design. In this paper we use ZEMAX [13]. In ZEMAX, optical surface deformation can be defined by a surface type called Zernike Standard Sag [18]. The Zernike Standard Sag surface is defined as an even aspheric surface plus aspheric terms defined by the Zernike Standard coefficients.

$$
z=\frac{c r^{2}}{1+\sqrt{1+(1+k) c^{2} r^{2}}}+\sum_{i=1}^{8} \alpha_{i} r^{2 i}+\sum_{i=1}^{N} a_{i} Z_{i}(\rho, \theta)
$$

where $\mathrm{c}$ is the curvature, $\mathrm{r}$ is the radial coordinate, $\mathrm{k}$ is the conic constant, $\alpha_{\mathrm{i}}$ is the even aspheric coefficient, and $\mathrm{N}$ is the number of Zernike coefficients in the series. The rigid body motions of mirrors or lens are expressed by the change of thickness along the optical axis, decenter and tilt of the elements. Tables 3 and 4 list sample data for

TABLE 3. Rigid body motions of the optical elements of the EO channel due to $1 \mathrm{~g}$ gravity along the $\mathrm{X}$ axis

\begin{tabular}{c|c|c|c|c|c}
\hline \hline & $\begin{array}{c}\text { Thickness change } \\
(\mu \mathrm{m})\end{array}$ & $\mathrm{X}$ & $\mathrm{Y}$ & \multicolumn{2}{c}{ Tilt (degree) } \\
\cline { 3 - 5 } & 0.00 & -0.760 & -0.020 & 0.000 & $\mathrm{Y}$ \\
\hline Splitter & 0.00 & -0.280 & 0.000 & 0.000 & 0.000 \\
\hline EO_Mr_1 & 0.00 & 0.340 & 0.000 & 0.000 & 0.000 \\
\hline EO_Lens_1 & 0.00 & 0.350 & 0.000 & -0.001 & 0.000 \\
\hline EO_Lens_2 & -0.360 & 0.000 & -0.002 & 0.004 \\
\hline EO_Lens_3 & 0.00 & -0.370 & 0.000 & 0.000 & 0.000 \\
\hline EO_Lens_4 & 0.00 & 0.440 & 0.000 & 0.000 & 0.000 \\
\hline EO_Mr_2 & 0.00 & 0.000 & 0.000 & 0.000 & 0.002 \\
\hline EO_Mr_3 & -0.40 & & & & \\
\hline
\end{tabular}

TABLE 4. Zernike polynomial coefficients or optical deformations of the IR channel optics due to $1 \mathrm{~g}$ gravity along the $\mathrm{Y}$ axis

\begin{tabular}{c|c|c|c|c|c|c|c|c}
\hline \hline & Splitter & IR_Lens_1 & IR_Lens_1 & IR_Lens_2 & IR_Lens_3 & IR_Lens_4 & IR_Lens_5 & IR_Lens_2 \\
\hline 1 & 37.464 & 6.358 & 9.262 & $80,142.200$ & $5,616.160$ & $-7,307.770$ & $-1,470.610$ & -140.137 \\
\hline 2 & 4.010 & -1.173 & -0.417 & -796.284 & -351.971 & 10.395 & -81.907 & -5.308 \\
\hline 3 & 60.085 & 54.379 & 45.112 & 281.674 & -37.286 & -138.696 & -2.168 & -42.398 \\
\hline 4 & -0.947 & 0.041 & -0.608 & -0.710 & 0.026 & 1.420 & -0.078 & 0.328 \\
\hline 5 & -0.212 & -0.774 & 0.006 & -1.640 & 1.233 & 0.247 & -0.057 & 0.002 \\
\hline 6 & 0.365 & -0.727 & 0.491 & -1.642 & -0.111 & 3.815 & -0.108 & -0.184 \\
\hline 7 & 0.024 & -0.078 & 0.136 & 0.000 & 0.000 & -0.003 & 0.000 & -0.036 \\
\hline 8 & 0.001 & -0.044 & 0.002 & 0.001 & -0.081 & -0.053 & -0.262 & -0.003 \\
\hline 9 & -0.124 & 0.040 & -0.061 & 0.001 & 0.000 & 0.005 & 0.000 & 0.033 \\
\hline 10 & 0.150 & -0.221 & -0.004 & 0.002 & -0.078 & -0.055 & -0.262 & -0.002 \\
\hline 11 & 0.095 & -0.005 & 0.066 & 0.000 & 0.000 & 0.000 & 0.000 & -0.064 \\
\hline 12 & -0.035 & -0.022 & -0.037 & 0.000 & 0.000 & 0.000 & 0.000 & 0.044 \\
\hline 13 & -0.019 & -0.018 & -0.001 & 0.000 & 0.000 & 0.000 & 0.000 & -0.001 \\
\hline 14 & 0.059 & 0.059 & 0.027 & 0.000 & 0.000 & 0.000 & 0.000 & -0.028 \\
\hline 15 & -0.061 & -0.026 & 0.000 & 0.000 & 0.000 & 0.000 & 0.000 & 0.001 \\
\hline 16 & 0.001 & 0.002 & -0.001 & 0.000 & 0.000 & 0.000 & 0.000 & 0.001 \\
\hline 17 & 0.008 & 0.002 & -0.025 & 0.000 & 0.000 & 0.000 & 0.000 & -0.015 \\
\hline 18 & -0.025 & 0.022 & 0.001 & 0.000 & 0.000 & 0.000 & 0.000 & 0.001 \\
\hline 19 & -0.011 & 0.028 & 0.011 & 0.000 & 0.000 & 0.000 & 0.000 & 0.006 \\
\hline 20 & -0.001 & 0.020 & -0.007 & 0.000 & 0.000 & 0.000 & 0.000 & -0.004 \\
\hline 21 & 0.016 & 0.050 & 0.000 & 0.000 & 0.000 & 0.000 & 0.000 & 0.000 \\
\hline
\end{tabular}


integrating FEA results with ZEMAX.

\subsection{Predicted Imaging Performance}

The modulation transfer function (MTF) is used as the performance metric in this paper, and refocus or the longitudinal shift of the image plane is applied to allow compensation. Fig. 9 shows the MTF variations of the EO channel for $1 \mathrm{~g}$ gravity along the $\mathrm{Z}$ axis. The imaging performance of the EO channel drops by $9 \%$ and then back to the design value with refocus. Both the EO and IR channels are simulated to keep the design performance with refocus capability regardless of the gravity direction. Fig. 10 shows the MTF variations of the EO channel for the temperature gradient across the y axis $\left(\Delta \mathrm{T}=10^{\circ} \mathrm{C}\right)$. The EO channel shows about a $20 \%$ MTF drop after the surface deformations are considered, which means that some optical surfaces are distorted. However, the distortion is shown to mostly be compensated for by refocusing the focal plane. Unlike the EO channel, the IR channel does not show any significant change.

Figs. 11 and 12 show the MTF variation of the EO and IR channels for the overall temperature change $\left(\Delta \mathrm{T}=20^{\circ} \mathrm{C}\right)$. The EO and IR channels experience a significant performance drop due to the overall temperature change of the camera before applying refocus. The performance drop is worse in the EO channel than in the IR channel. Those results require that the EO/IR camera be refocused on air, and an application of active thermal control is desirable.

Table 5 summarizes the predicted MTF at the Nyquist frequency of each detector. The Nyquist frequencies for the EO and IR channels are 27.3 and 13.0 line-pairs-per-mm (lpm), respectively. First, the IR channel can sustain imaging perfor-
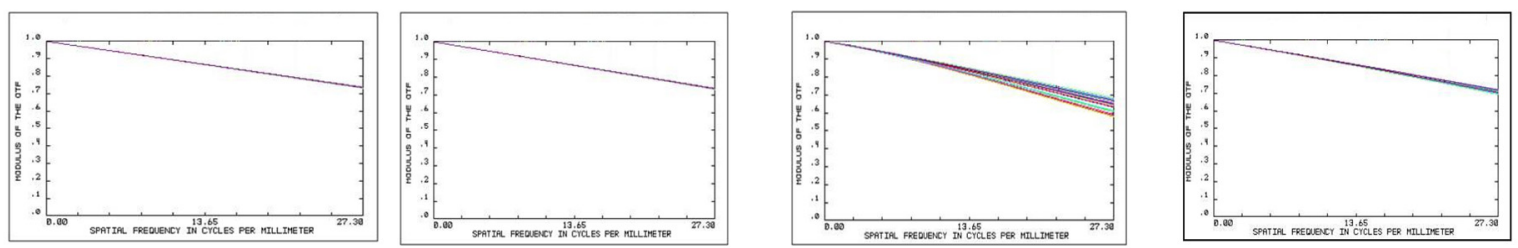

FIG. 9. MTF variation of the EO channel as the FEA results are considered and then refocused due to $1 \mathrm{~g}$ gravity along the $\mathrm{Z}$ axis.
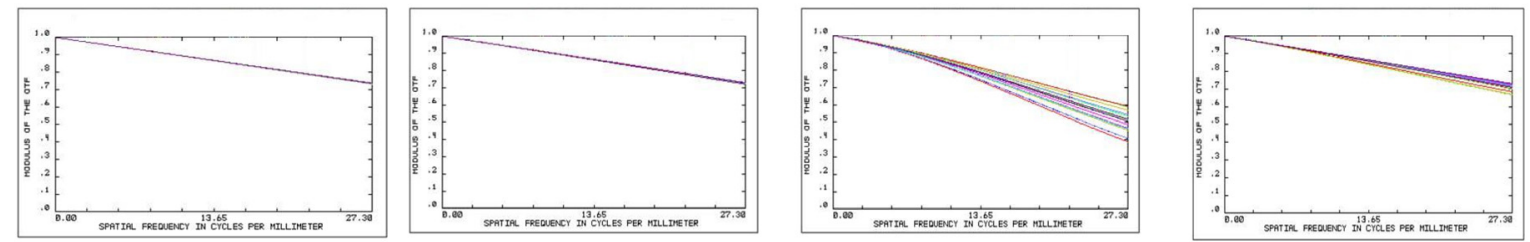

FIG. 10 MTF variation of the EO channel as the FEA results are considered and refocused due to the temperature gradient across y axis $\left(\Delta \mathrm{T}=10^{\circ} \mathrm{C}\right)$.
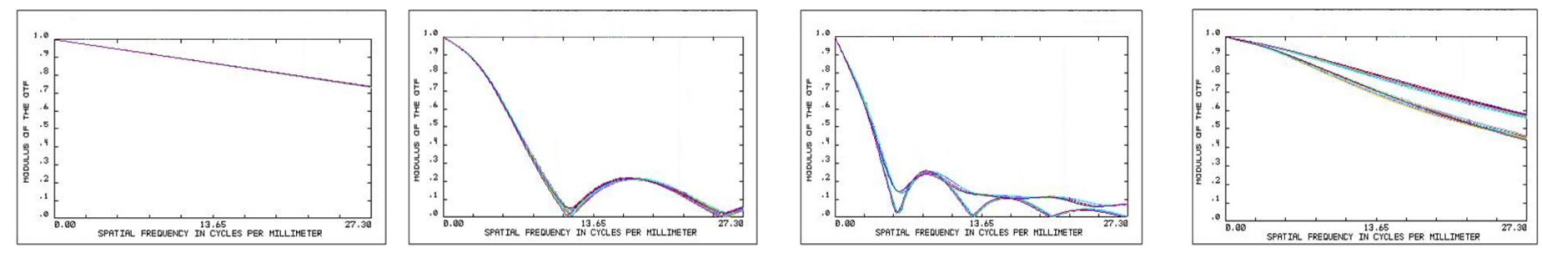

FIG. 11. MTF variation of the EO channel as the FEA results are considered and refocused due to the overall temperature change $\left(\Delta \mathrm{T}=20^{\circ} \mathrm{C}\right)$.
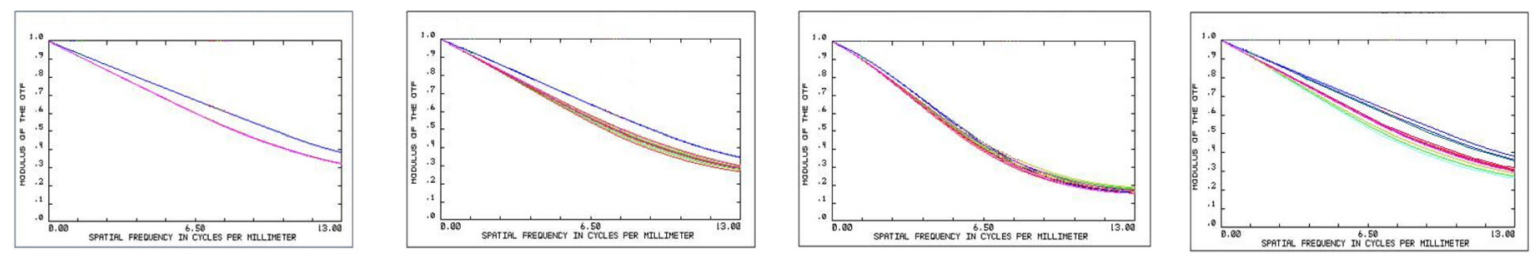

FIG. 12. MTF variation of the IR channel as the FEA results are considered and refocused due to the overall temperature change $\left(\Delta \mathrm{T}=20^{\circ} \mathrm{C}\right)$. 
TABLE 5. Predicted imaging performance

\begin{tabular}{|c|c|c|c|c|c|c|}
\hline \multirow{2}{*}{\multicolumn{3}{|c|}{ Case }} & \multicolumn{3}{|c|}{ MTF at Nyquist frequency } & \multirow{3}{*}{$\begin{array}{c}\text { Refocus }(\mu \mathrm{m}) \\
7.2\end{array}$} \\
\hline & & & Design & Before refocus & After refocus & \\
\hline \multirow{5}{*}{$\begin{array}{c}\text { EO } \\
\text { channel }\end{array}$} & \multirow{3}{*}{$\begin{array}{l}\text { Gravity } \\
\text { effects }\end{array}$} & 1g along $\mathrm{x}$ & \multirow{5}{*}{0.74} & 0.73 & 0.74 & \\
\hline & & $1 \mathrm{~g}$ along $\mathrm{y}$ & & 0.74 & 0.74 & 0.0 \\
\hline & & $1 \mathrm{~g}$ along $\mathrm{z}$ & & 0.65 & 0.72 & 80.6 \\
\hline & \multirow{2}{*}{$\begin{array}{l}\text { Thermal } \\
\text { effects }\end{array}$} & $\begin{array}{c}\text { Temperature gradient } \\
\text { across y axis }\left(\Delta T=10^{\circ} \mathrm{C}\right)\end{array}$ & & 0.51 & 0.71 & 141.1 \\
\hline & & Overall temperature change $\left(\Delta \mathrm{T}=20^{\circ} \mathrm{C}\right)$ & & 0.00 & 0.59 & 1010.0 \\
\hline \multirow{5}{*}{$\begin{array}{c}\text { IR } \\
\text { channel }\end{array}$} & \multirow{3}{*}{$\begin{array}{l}\text { Gravity } \\
\text { effects }\end{array}$} & $1 \mathrm{~g}$ along $\mathrm{x}$ & \multirow{5}{*}{0.38} & 0.38 & 0.38 & 0.0 \\
\hline & & $1 \mathrm{~g}$ along $\mathrm{y}$ & & 0.25 & 0.38 & 354.9 \\
\hline & & $1 \mathrm{~g}$ along $\mathrm{z}$ & & 0.38 & 0.38 & 24.3 \\
\hline & \multirow{2}{*}{$\begin{array}{l}\text { Thermal } \\
\text { effects }\end{array}$} & $\begin{array}{c}\text { Temperature gradient } \\
\text { across y axis }\left(\Delta \mathrm{T}=10^{\circ} \mathrm{C}\right)\end{array}$ & & 0.36 & 0.38 & 152.3 \\
\hline & & Overall temperature change $\left(\Delta \mathrm{T}=20^{\circ} \mathrm{C}\right)$ & & 0.17 & 0.38 & 505.1 \\
\hline
\end{tabular}

mance as good as designed with refocus capability for all gravitation and thermal conditions. The EO channel experiences slight performance degradation (16\% MTF drop) for a $20^{\circ} \mathrm{C}$ overall temperature change. This result might require the EO/IR camera to be actively thermal controlled.

\section{CONCLUSION}

This paper reports an integrated performance prediction of an EO/IR dual band camera for an AV. The camera is basically a Ritchey-Chrétien telescope with $\sim 30 \mathrm{~cm}$ entrance pupil diameter with relay optics delivering visible and IR images to the detectors (a CCD and an IR detector).

The reported EO/IR camera is shown by finite element analysis to be mechanically stiff over any directional gravitational force and two thermal conditions. The two thermal conditions are overall temperature change to $30^{\circ} \mathrm{C}$ from $20^{\circ} \mathrm{C}$ and temperature gradient across the y axis from $-5^{\circ} \mathrm{C}$ to $+5^{\circ} \mathrm{C}$.

An investigation over the MTF variation of the camera is performed by integrating the FEA results into lens design/analysis software ZEMAX. The analysis shows both the EO and IR channels mostly keep imaging performance as good as designed with refocus capability. However, the EO channel is shown to be slightly susceptible to overall temperature change and it is expected to experience slight performance degradation from $74 \%$ to $59 \%$ in terms of MTF for $20^{\circ} \mathrm{C}$ overall temperate change. Further investigation will be performed over active thermal control on the EO/IR camera.

\section{ACKNOWLEDGMENT}

This study is funded by Agency for Defense Development.

\section{REFERENCES}

1. R. A. Schowengerdt, Remote Sensing (Academic Press, USA, 2007), Chapter 1.

2. C. Cox, S. Kishner, and R. Whittesy, "Reconnaissance payloads for responsive space," AIAA-3rd Responsive Space Conference (2005).

3. D. Zhang, G. Ma, and H. Liu, "Aerial reconnaissance camera using optical drum and mirror rotation mechanicsm," in Proc. Systems and Control in Aeronautics and Astronautics (Harbin, China, 2010), pp. 415-418.

4. J. H. Lee, T. S. Jang, H.-S. Yang, and S.-W. Rhee, "Optical design of a compact imaging spectrometer for STSAT3," J. Opt. Soc. Korea 12, 262-268 (2008).

5. M. Stanton, W. C. Phillips, Y. Li, and K. Kalata, "The detective quantum efficiency of CCD and vidicon-based detectors for X-ray crystallographic applications," J. Appl. Cryst. 25, 638-645 (1992).

6. http://www.e2v.com.

7. http://www.sofradir.com.

8. M. Henini and M. Razeghi, Handbook of Infrared Detection Technologies (Elsevier Science Ltd., Oxford, UK, 2002), Chapter 2.

9. S. R. Beran, A. J. Partynski, A. M. Baker, and J. Jones, "Cassegrain optical system for framing aerial reconnaissance camera," US patent 6374047 (2002).

10. V. Petrushevsky, Y. Karklinsky, and A. Chernobrov, "ELOp EO/IR LOROP camera: image stabilization for dual-band whiskbroom scanning photograpy," Proc. SPIE 4820, 607-617 (2003).

11. S. Seong, J. Yu, D. Ryu, J. Hong, J.-Y. Yoon, S.-W. Kim, J.-H. Lee, and M.-J. Shin, "Imaging and radiometric performance simulation for a new high performance dual band airborne reconnaissance camera," Proc. SPIE 7307, 730705-1 730705-11 (2009).

12. Y. An, C.-P. Song, R.-J. Kuang, and G. Jin, "Research on the device of non-angular vibration for opto-eltronic platform," Proc. SPIE 7659, 76590H-1 76590H-6 (2010). 
13. http://www.zemax.com.

14. R. J. Noll, "Zernike polynomials and atmospheric turbulence," J. Opt. Soc. Am. 66, 207-211 (1976).

15. N. Roddier, "Atmospheric wavefront simulation and Zernike polynomials," Proc. SPIE 1237, 668-679 (1990).

16. W. J. Smith, Modern Lens Design (McGraw-Hill, USA, 2005), Chapter 23.
17. T. Sasak, M. Shinkai, K. Higashiyama, F. Tanaka, and T. Kishinami, "Development of statistical tolerancing system for optical product - virtual PT system and mass product simulation," Proc. SPIE 3482, 528-537 (1998).

18. ZEMAX Optical Design Program User's Guide (ZEMAX Development Corporation, USA, 2005), Chapter 11. 